\title{
Trehalose is required for the acquisition of tolerance to a variety of stresses in the filamentous fungus Aspergillus nidulans
}

\author{
Sabine Fillinger, ${ }^{1}$ Marie-Kim Chaveroche, ${ }^{1}$ Patrick van Dijck, ${ }^{2}$ \\ Ronald de Vries, ${ }^{3}$ George Ruijter, ${ }^{3}$ Johan Thevelein ${ }^{2}$ \\ and Christophe d'Enfert ${ }^{1}$
}

\footnotetext{
1 Unité Microbiologie et Environnement, Institut Pasteur, 25 rue du Docteur Roux, 75724 Paris Cedex 15 , France

2 Flanders Interuniversity Institute for Biotechnology, VIB and Laboratory of Molecular Cell Biology, Katholieke Universiteit Leuven, Kardinaal Mercierlaan 92, B-3001 Leuven-Heverlee, Flanders, Belgium

3 Molecular Genetics of Industrial Micro-organisms, Wageningen University, Dreijenlaan 2, 6703HA Wageningen, The Netherlands
}

Author for correspondence: Christophe d'Enfert. Tel: +331406132 57. Fax: +3314568 8790 . e-mail: denfert@pasteur.fr
Trehalose is a non-reducing disaccharide found at high concentrations in Aspergillus nidulans conidia and rapidly degraded upon induction of conidial germination. Furthermore, trehalose is accumulated in response to a heat shock or to an oxidative shock. The authors have characterized the $A$. nidulans tpsA gene encoding trehalose-6-phosphate synthase, which catalyses the first step in trehalose biosynthesis. Expression of tpsA in a Saccharomyces cerevisiae tps1 mutant revealed that the tpsA gene product is a functional equivalent of the yeast Tps1 trehalose-6-phosphate synthase. The $A$. nidulans tpsA-null mutant does not produce trehalose during conidiation or in response to various stress conditions. While germlings of the tpsA mutant show an increased sensitivity to moderate stress conditions (growth at $45^{\circ} \mathrm{C}$ or in the presence of $2 \mathrm{mM} \mathrm{H}_{2} \mathrm{O}_{2}$ ), they display a response to severe stress (60 $\mathrm{min}$ at $50{ }^{\circ} \mathrm{C}$ or in the presence of $100 \mathrm{mM} \mathrm{H}_{2} \mathrm{O}_{2}$ ) similar to that of wild-type germlings. Furthermore, conidia of the tpsA mutant show a rapid loss of viability upon storage. These results are consistent with a role of trehalose in the acquisition of stress tolerance. Inactivation of the tpsA gene also results in increased steady-state levels of sugar phosphates but does not prevent growth on rapidly metabolizable carbon sources (glucose, fructose) as seen in Saccharomyces cerevisiae. This suggests that trehalose 6-phosphate is a physiological inhibitor of hexokinase but that this control is not essential for proper glycolytic flux in A. nidulans. Interestingly, tpsA transcription is not induced in response to heat shock or during conidiation, indicating that trehalose accumulation is probably due to a post-translational activation process of the trehalose 6-phosphate synthase.

Keywords: trehalose 6-phosphate synthase, spore germination, glycolysis, heat stress, oxidative stress, hexokinase

\section{INTRODUCTION}

Trehalose ( $\alpha$-D-glucopyranosyl- $\alpha$-D-glucopyranoside) is a non-reducing disaccharide found in a wide variety of organisms (bacteria, fungi, protozoa, plants). In lower eukaryotes, it constitutes up to $15 \%$ of the dry weight of

Abbreviations: EST, expressed sequence tag; 5-FOA, 5-fluoro-orotic acid; T6P, trehalose 6-phosphate; T6PP, trehalose-6-phosphate phosphatase; T6PS, trehalose-6-phosphate synthase.

The GenBank accession number for the sequence reported in this paper is AF043230. stationary-phase cells or spores (Elbein, 1974). The universal mobilization of trehalose during growth resumption from resting stages supports a role as a storage carbohydrate (Arguelles, 2000; Thevelein, 1984, 1996). However, because of a number of physico-chemical properties, including high hydrophilicity and chemical stability, non-hygroscopic glass formation and the absence of internal hydrogen bond formation, trehalose is apparently able to serve a unique role as stress metabolite (Arguelles, 2000; Thevelein, 1996).

In the yeast Saccharomyces cerevisiae, biosynthesis of trehalose is mediated by a multi-protein complex that 


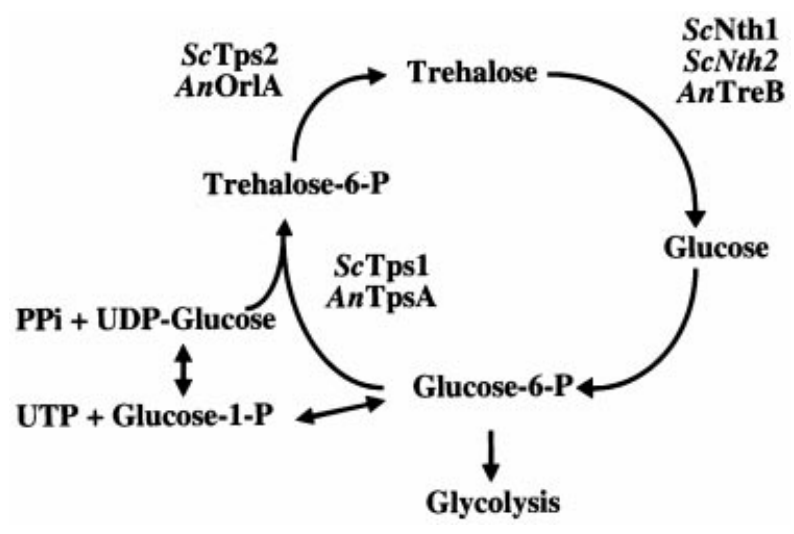

Fig. 1. Trehalose metabolism in fungi. ScTps 1 and $A n T p s A$, trehalose-6-phosphate synthase; ScTps2 and AnOrIA, trehalose6-phosphate phosphatase; ScNth1, ScNth2 and AnTreB, neutral trehalase; Sc, S. cerevisiae; An, A. nidulans.

contains a trehalose-6-phosphate synthase (T6PS; Fig. 1) encoded by TPS1 and a trehalose-6-phosphate phosphatase (T6PP; Fig. 1) encoded by TPS2 (Bell et al., 1992, 1998; de Virgilio et al., 1993). This multi-protein complex contains regulatory subunits, the products of the redundant TSL1 and TPS3 genes, which share a conserved amino-terminal domain with Tps1 and Tps2 (Bell et al., 1998; Reinders et al., 1997; Vuorio et al., 1993). Mobilization of the trehalose pool in response to various stimuli is mediated by a neutral trehalase encoded by NTH1 (Kopp et al., 1993) while the role of a second neutral trehalase encoded by NTH2 remains uncertain (Nwaka et al., 1995). Studies using mutants in the different genes of trehalose metabolism in S. cerevisiae and in other yeasts support the protective role of trehalose and more specifically a role in the acquisition of stress tolerance (Arguelles, 2000). However, additional functions have been proposed for trehalose 6phosphate (T6P), which is the first intermediate in the biosynthesis of trehalose (Fig. 1). In particular, analysis of $S$. cerevisiae mutants impaired in the biosynthesis of T6P has indicated a role for T6P and the T6PS in the control of the influx of glucose into glycolysis (Blazquez et al., 1993; Bonini et al., 2000; Hohmann et al., 1996; Thevelein \& Hohmann, 1995).

Several studies have been conducted to determine the role of trehalose and T6P in filamentous fungi but they have remained inconclusive. Two genes encoding T6PS have been identified in Aspergillus niger (Wolschek \& Kubicek, 1997). However, disruption of only one of these genes has been achieved and therefore an unambiguous role for T6P or trehalose in filamentous fungi could not be deduced from this study (Wolschek \& Kubicek, 1997). Arisan-Atac et al. (1996) have nevertheless shown that T6P participates in the control of citrate production in A. niger. In A. nidulans, T6PP is encoded by the orlA gene, the inactivation of which results in an osmoremediable thermosensitive growth phenotype that can be explained by an inhibitory role of
T6P in the control of chitin biosynthesis (Borgia et al., 1996). However, orlA mutants produce normal levels of trehalose and are therefore not suited to determine the role of trehalose in filamentous fungi (Borgia et al., 1996). T6P is a potent inhibitor of hexokinase also in $A$. nidulans and $A$. niger, suggesting a role in the control of glycolytic flux in these species (Panneman et al., 1998; Ruijter et al., 1996). Finally, neutral trehalases have also been identified in filamentous fungi and a role in the control of trehalose mobilization has been demonstrated during growth resumption stages, in particular spore germination, in a manner similar to that observed in yeast (d'Enfert et al., 1999).

Interestingly, analysis of A. nidulans mutants devoid of neutral trehalase has suggested that trehalose has a minor role as a storage carbohydrate and could function in the protection of germinating conidia against thermal stress (d'Enfert et al., 1999).

Here, we report the characterization of the $t p s A$ gene of A. nidulans, encoding a T6PS, and the construction of an $A$. nidulans tpsA-null mutant. This mutant fails to accumulate trehalose in response to a variety of stress conditions but this defect is not associated with an increased sensitivity to a short exposure of the same stress conditions. In contrast, this mutant is defective for growth at high temperature, shows an increased sensitivity to long exposure to sublethal doses of reactive oxygen species and has reduced spore viability, thus suggesting a role for trehalose in the resistance of $A$. nidulans to sustained exposure to various stress conditions, including starvation.

\section{METHODS}

Strains and growth conditions. A. nidulans strains FGSC28 (pabaA6 biA1) and FGSC773 (wA3; pyroA4; pyrG89) were obtained from the Fungal Genetics Stock Centre (University of Kansas, Kansas City, KA, USA). A. nidulans strains CEA150 ( wA3; pyroA4; pyrG89; tpsA-AfpyrG-tpsAs) and CEA152 ( $w A 3$; pyroA4; pyrG89; tpsA $\Delta)$ are derivatives of FGSC773 obtained in the course of this study. Growth conditions for $A$. nidulans strains have been described (d'Enfert \& Fontaine, 1997). $\mathrm{H}_{2} \mathrm{O}_{2}$ was added to solid culture media in a $0-5 \mathrm{mM}$ range. Cultures of $A$. nidulans strains for the assay of heat or oxidative-shock sensitivity were inoculated at $2 \times 10^{7}$ conidia $\mathrm{ml}^{-1}$ and grown for $3 \mathrm{~h}$ at $30{ }^{\circ} \mathrm{C}$ in minimal glucose medium containing $0.01 \%$ Tween-20. Following heat shock $\left(0-60 \mathrm{~min}\right.$ at $\left.50^{\circ} \mathrm{C}\right)$ or oxidative shock $\left(100 \mathrm{mM} \mathrm{H}_{2} \mathrm{O}_{2}\right.$ for 0-60 min), an aliquot of each culture was withdrawn and serially diluted in PBS $(150 \mathrm{mM} \mathrm{NaCl}, 10 \mathrm{mM}$ sodium phosphate $\mathrm{pH} 7 \cdot 2) / 0 \cdot 1 \%$ Tween-20. Heat- or $\mathrm{H}_{2} \mathrm{O}_{2}$-shocked cells were then plated on complete medium containing $0 \cdot 1 \%$ Triton X-100 in order to limit the growth of the colonies. Colonies were counted after a $2 \mathrm{~d}$ incubation at $37^{\circ} \mathrm{C}$. The same cultures were used to measure trehalose content in the conidia with previously described procedures (d'Enfert \& Fontaine, 1997). Alternatively, trehalose, T6P, sugar phosphates and ATP were measured using extracts from mycelia grown at $30{ }^{\circ} \mathrm{C}$ in minimal glucose medium for $4 \mathrm{~h}$ using the procedure of Ruijter \& Visser (1996). Conidium viability was monitored using duplicates of two independent stocks of FGSC773 and CEA152 conidia stored at either $4{ }^{\circ} \mathrm{C}$ or $20^{\circ} \mathrm{C}$ in 
Table 1. Oligonucleotides used in this study

\begin{tabular}{|ll|}
\hline Oligonucleotide & \multicolumn{1}{c|}{ Sequence } \\
\hline tps1F & $5^{\prime}$-TGGCCNCTNTTCCAYTACCA-3' \\
tps1B & $5^{\prime}$-GGNACNCCYTTRATGTARTC-3' \\
tps5'Bgl & $5^{\prime}$-TGCAGATCTCCCGGCGTTGAGAAATCC-3' \\
tps3'Not & $5^{\prime}$-TTGATGCGGCCGCTACTGTGACGAAGTCTC-3' \\
tps4 & $5^{\prime}$-GTTGCGAGCCAAGTTCAG-3' \\
tps5 & $5^{\prime}$-CCCTGGAATTCTATCCCA-3' \\
tps6 & $5^{\prime}$-CATTGTGATGTTGAACA-3' \\
tps9 & $5^{\prime}$-GGTTGGCAGTCCCAGCAA-3' \\
tps10 & $5^{\prime}$-AGGAGATCCCGACTCTG-3' \\
tps11 & $5^{\prime}$-TACTCGGTCAGACCAGG-3' \\
\hline
\end{tabular}

PBS $/ 0 \cdot 1 \%$ Tween-20. At different time intervals, an aliquot of each stock was serially diluted and plated in duplicate on complete medium containing $0.01 \%$ Triton X-100. Colonies were counted after a $2 \mathrm{~d}$ incubation at $37^{\circ} \mathrm{C}$. Conidiospore germination was monitored by microscopic examination of slides coated with minimal glucose medium and spot-inoculated with approximately $10^{4}$ freshly harvested conidia. The percentage of germinated spores was recorded at different times.

The $S$. cerevisiae strains used in this study were W303.1A (Thomas \& Rothstein, 1989; MATa leu2-3,112 ura3-1 trp1-92 his3-11,15 ade2-1 can1-100 GAL SUC mal) and the isogenic YSH290 strain containing the tps1s mutation (Neves et al., $1995)$. Yeast cells were routinely grown on a rotary shaker at $30{ }^{\circ} \mathrm{C}$ in yeast nitrogen base medium (YNB; Sherman, 1991) containing $2 \%$ glucose or $2 \%$ galactose (YSH290) as carbon source.

Escherichia coli strains PAP105 $\left[\Delta\right.$ (lac-pro) $\quad \mathrm{F}^{\prime}\left(\right.$ lacl $^{\mathrm{q} 1}$ $\Delta\left(\right.$ lacZ)M15 pro $\left.\left.^{+} \operatorname{Tn} 10\right)\right]$ and DH5 $\alpha$ (Woodcock et al., 1989) were used for plasmid propagation. The $\beta$-lactam antibiotic carbenicillin $\left(100 \mu \mathrm{g} \mathrm{ml}^{-1}\right)$ and tetracycline $\left(15 \mu \mathrm{g} \mathrm{ml}^{-1}\right)$ were added to the growth medium when required.

PCR amplification of a segment of the $A$. nidulans tpsA gene. The genomic DNA of $A$. nidulans FGSC28 prepared according to Girardin et al. (1993) was used as template to amplify a segment of genes potentially encoding a T6PS. The sense and antisense primers (tps1F and tps1B, Table 1) were based on amino-acid sequences (WPLFHYH and DYIKGVP, respectively) conserved in several fungal T6PS (Bell et al., 1992; Blazquez et al., 1994; Luyten et al., 1993; Wolschek \& Kubicek, 1997). The amplification protocol consisted of a denaturation step at $94{ }^{\circ} \mathrm{C}$ for 5 min followed by 35 cycles of the following steps: denaturation at $94^{\circ} \mathrm{C}$ for $30 \mathrm{~s}$, annealing at $55^{\circ} \mathrm{C}$ for $1 \mathrm{~min}$, extension at $72{ }^{\circ} \mathrm{C}$ for $2 \mathrm{~min}$. A last elongation step was carried out at $72{ }^{\circ} \mathrm{C}$ for $10 \mathrm{~min}$. An approximately $650 \mathrm{bp}$ amplification product was gel purified and cloned in Bluescript $\mathrm{SK}^{+}$(Stratagene) using standard cloning procedures. Two plasmids, pTPS1 and pTPS2, were obtained that carry the amplification product in opposite orientations.

DNA and RNA manipulations. General recombinant DNA techniques and Southern-blot analyses were essentially performed according to Sambrook et al. (1989) and Ausubel et al. (1992). Transformation of calcium-manganese-treated E. coli was as described by Hanahan et al. (1991). Oligonucleotides used in this study were obtained from Genset (Paris, France) and are listed in Table 1. A BamHI-EcoRV fragment corresponding to the cloned PCR product was labelled with $\left[\alpha^{-32} \mathrm{P}\right] \mathrm{dCTP}$ using the Megaprime kit (Amersham) and used to probe a replica of a genomic library (a gift from C. Scazzochio) made in plasmid pFB39, a derivative of pUC18 carrying the $A$. nidulans $\arg B$ gene. This screening yielded a single positive plasmid, pTPS4, which lacked the $3^{\prime}$ end of the tps $A$ gene as deduced from DNA sequencing. To identify a clone carrying the entire tps $A$ gene, the chromosome-specific libraries of A. nidulans genomic DNA (Brody et al., 1991) that had been obtained from the Fungal Genetic Stock Center and transferred onto nylon membranes (ZetaProbe, Bio-Rad) were probed using a $400 \mathrm{bp} \mathrm{EcoRI} \mathrm{fragment} \mathrm{located} \mathrm{at} \mathrm{the} 3^{\prime}$ end of the cloned tpsA region and labelled as above. The plasmids pTPS6 and pTPS7 are derivatives of pUC18 and pBLSN ${ }^{+}$ (d'Enfert, 1996), respectively, that carry a $1.75 \mathrm{~kb}$ SalI-SmaI fragment containing the region of $t p s A$ carried by pTPS4. The plasmids pTPS11 and pTPS12 are derivatives of pBLSN ${ }^{+}$ which carry a $2.4 \mathrm{~kb}$ EcoRI fragment of cosmid L24E04 in opposite orientations. DNA sequencing was performed by the dideoxy chain termination method (Sanger et al., 1977) on double-stranded plasmids derived from pTPS6, pTPS7, pTPS11 and pTPS12 by internal restriction-enzyme-mediated deletions and using a set of appropriate oligonucleotide primers. The sequence of a $2699 \mathrm{bp}$ fragment was read at least twice on each strand and is deposited in the GenBank database under accession number AF043230.

Plasmid pTPS13 was obtained by subcloning the $0.8 \mathrm{~kb}$ SalI-ClaI fragment of pTPS7 into SalI-ClaI-digested pTPS$11 \Delta \mathrm{P}$, a derivative of $\mathrm{pTPS} 11$ with an internal deletion of a Pst $\mathrm{I}$ fragment. Plasmid pTPS17 was then obtained by subcloning a $K s p \mathrm{I}-E c o \mathrm{RV}$ fragment carrying the A. fumigatus pyrG gene (Weidner et al., 1998) into KspI/SmaI-digested pTPS13. Plasmid pTPS17 was used to transform protoplasts of $A$. nidulans strains (Osmani et al., 1987). Genomic DNA from 15 prototrophic transformants was prepared according to Mol et al. (1998) and screened by PCR using primers tps10 and tps11 (Table 1). While a $2006 \mathrm{bp}$ product is expected in transformants carrying an ectopic integration of pTPS17 or a tpsAs-AfpyrG-tpsA allele, a $1462 \mathrm{bp}$ fragment is expected in transformants with a $t p s A-p y r G-t p s A \Delta$ allele. Putative merodiploids with a $t p s A-A f p y r G-t p s A \Delta$ allele were confirmed by Southern blot analysis of EcoRI/SalI-digested genomic DNA prepared according to Girardin et al. (1993) and probed with a $0.8 \mathrm{~kb}$ ClaI-SalI fragment of pTPS13 that had been labelled with the Rediprime labelling kit (Amersham). Washed membranes were exposed to X-omat films (Kodak). While strains carrying only a wild-type tps $A$ allele show a $1.15 \mathrm{~kb}$ hybrid-

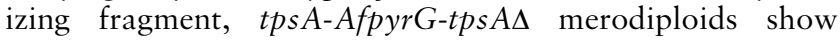


$1 \cdot 15 \mathrm{~kb}$ and $0.8 \mathrm{~kb}$ hybridizing fragments corresponding to the wild-type and mutant allele respectively. Conversion of the tpsA-AfpyrG-tpsA $\Delta$ allele to the $t p s A \Delta$ allele was obtained by plating conidia of strain CEA150 on minimal glucose plates containing $1 \mathrm{mg} \mathrm{ml}^{-1}$ 5-fluoro-orotic acid (5-FOA), uridine, uracil and pyridoxine. $\mathrm{HCl}$, thus promoting the excision of the A. fumigatus pyrG gene through recombination between the two tpsA alleles (d'Enfert \& Fontaine, 1997). The nature of the tps $A$ allele in 5-FOA-resistant clones was checked by PCR using primers tps10 and tps11 (Table 1), which can discriminate between wild-type tps $A$ and tps $A \Delta$, and with primers tps 4 and tps5 (Table 1), which yield only a $363 \mathrm{bp}$ product if the wild-type tpsA gene is present. Excision of the A. fumigatus pyrG gene was confirmed by Southern blot analysis as described above. In this case a single EcoRI-SalI fragment could be detected corresponding to either the wildtype allele $(1.15 \mathrm{~kb})$ or the mutant tps $A \Delta$ allele $(0.8 \mathrm{~kb})$.

Preparation of total RNA from conidia, germinating conidia, heat-shocked germinating conidia, mycelia and developing cultures of A. nidulans strain pabaA1 and FGSC773 has been previously reported (d'Enfert et al., 1999). RT-PCR experiments were achieved using the Reverse Transcription System according to the manufacturer's instructions (Promega). Approximately $1 \mu \mathrm{g}$ total RNA was used for each oligo-dT primed reverse transcription. An aliquot of the reaction was then subjected to the following amplification protocol using primers tps 4 and tps5 (Table 1): a denaturation step at $93.5^{\circ} \mathrm{C}$ for $5 \mathrm{~min}$ followed by 20 cycles of the following steps: denaturation at $93.5{ }^{\circ} \mathrm{C}$ for $30 \mathrm{~s}$, annealing at $58^{\circ} \mathrm{C}$ for $1 \mathrm{~min}$, extension at $71^{\circ} \mathrm{C}$ for $1 \mathrm{~min}$. Amplification was limited to 20 or 25 cycles in order to remain in a linear range and therefore produce semi-quantitative data. The tps 4 and tps 5 oligonucleotides are sense and anti-sense primers, respectively, that are located on both sides of an intron in the tps $A$ gene. Therefore amplification from genomic DNA yields a $363 \mathrm{bp}$ product while amplification from reverse-transcribed mRNA yields a 308 bp product. Alternatively, primers tps 4 and tps 9 were used that yield a $282 \mathrm{bp}$ fragment corresponding only to reverse-transcribed mRNA because tps9 overlaps with an intron in $t p s A$.

Expression of tpsA in $\boldsymbol{S}$. cerevisiae. To obtain a cDNA encompassing the full tpsA ORF, total RNA prepared from the mycelium of a $A$. nidulans pabaA1 strain was reverse transcribed as described above. Reverse transcription products were then amplified using primers tps5'Bgl and tps3'Not (Table 1) and the following amplification procedure. A denaturation step at $93^{\circ} \mathrm{C}$ for $5 \mathrm{~min}, 30$ cycles of the following steps: denaturation at $93{ }^{\circ} \mathrm{C}$ for $30 \mathrm{~s}$, annealing at $54{ }^{\circ} \mathrm{C}$ for $1 \mathrm{~min}$, extension at $72{ }^{\circ} \mathrm{C}$ for $5 \mathrm{~min}$ and a final extension step of $10 \mathrm{~min}$ at $72{ }^{\circ} \mathrm{C}$. The amplification product was subcloned using the TA cloning kit according to the supplier's instructions (Invitrogen), yielding plasmid pTPS15. Following sequencing of the cloned tpsA cDNA, the BglII-NotI fragment of pTPS15 was subcloned into the yeast expression vector pCM190L (Llorente et al., 1999) cut by BamHI and NotI. Controlled expression in yeast is achieved by a tetracyclinerepressible promoter. Furthermore, the protein is produced as a fusion with an HA-epitope and a (His) ${ }_{6}$ tail that allows quantification of protein production. Two independent recombinants, pTPS16-1 and pTPS16-2, were selected for transformation into the $S$. cerevisiae strain YSH290 along with pCM190L and pCM190L::X, a derivative of pCM190L carrying a $S$. cerevisiae ORF unlinked to trehalose metabolism. Yeast transformation was performed using the one-step method (Chen et al., 1992). Trehalose levels in the transformants were measured using cells that had been grown into stationary phase on galactose as described by Neves et al. (1994).

\section{RESULTS}

\section{Isolation of the $A$. nidulans tps $A$ gene encoding a T6PS}

To investigate the role of trehalose in A. nidulans, we set out to identify genes encoding T6PS using a two-step strategy. First, two degenerate primers, tps1F (sense primer; Table 1) and tps1B (antisense primer; Table 1), were designed that correspond to two regions conserved in the T6PS of yeast (Bell et al., 1992; Blazquez et al., 1994; Luyten et al., 1993) and in the A. niger TpsA T6PS (Wolschek \& Kubicek, 1997). Using these primers, a 624 bp fragment was amplified by PCR from genomic DNA of $A$. nidulans strain FGSC28. The nucleotide sequence of this PCR product was determined and the deduced amino acid sequence revealed significant identity to corresponding regions of $A$. niger and $S$. cerevisiae T6PS $(94.2 \%$ and $66.8 \%$ identical amino acids, respectively). These results suggested that the PCR product corresponded to an A. nidulans T6PS-encoding gene referred to as tpsA.

In a second step, the PCR product was used to probe different libraries of $A$. nidulans genomic DNA. Using chromosome-specific libraries (Brody et al., 1991), three positive cosmids, L09H05, L14G06 and L24E04, were identified. These cosmids have been assigned to the same region of $A$. nidulans chromosome V (Prade et al., 1997), suggesting that the tps $A$ gene is located on this chromosome.

\section{Sequencing of the $A$. nidulans tps $A$ gene}

DNA sequencing of an approximately $1.8 \mathrm{~kb}$ SalI-SmaI fragment derived from plasmid pTPS4 (see Methods) and of an approximately $1.2 \mathrm{~kb}$ EcoRI-PstI fragment derived from cosmid L24E04 yielded a nucleotide sequence of 2699 bp (GenBank accession number AF043230; data not shown). Analysis of this DNA sequence revealed an ORF of $1512 \mathrm{bp}$ interrupted by four putative introns of 63, 55, 48 and 55 bp, respectively. The location of these introns was confirmed by sequencing a cDNA of tps $A$ obtained by amplification of reverse-transcribed mRNAs using primers tps $5^{\prime} \mathrm{Bgl}$ and tps $3^{\prime}$ Not (Table 1 ). Furthermore, analysis of several A. nidulans-expressed sequence tags (c3e03, m7e02, c5f08) determined within the $A$. nidulans EST sequencing program (D. Kupfer \& B. Roe, http://www.genome.ou.edu/fungal.html) confirmed the location of these introns and revealed the occurrence of an additional intron of $200 \mathrm{bp}$ located in the $5^{\prime}$-untranslated region of the gene and extending from position -302 to -103 relative to the $t p s A$ start codon (data not shown).

The A. nidulans 1512 bp ORF identified in the cloned DNA region encodes a 504 amino acid protein with a molecular mass of $56.8 \mathrm{kDa}$. This protein shares a minimum of $62 \cdot 2 \%$ identical amino acids and $74 \cdot 1 \%$ similar amino acids with known fungal T6PSs and its 


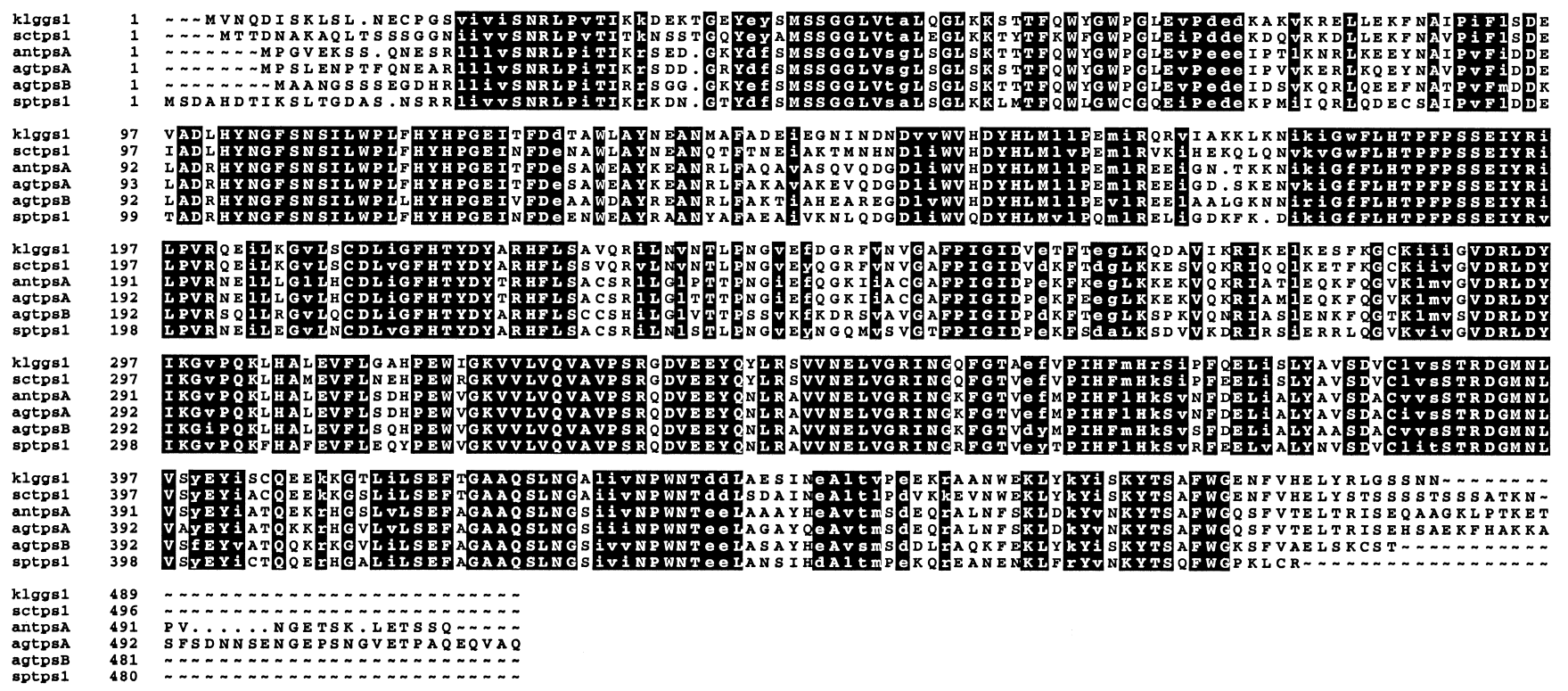

Fig. 2. Alignments for maximal amino acid similarities of the $A$. nidulans TpsA protein (antpsA) with the $S$. cerevisiae (sctps1; Bell et al., 1992), K. lactis (klggs1; Luyten et al., 1993), Schiz. pombe (sptps1; Blazquez et al., 1994) and A. niger (AgtpsA and AgtpsB; Wolschek \& Kubicek, 1997) T6PSs. This alignment was produced using the PILEUP program of the UWGCG package version 9 (Devereux et al., 1984). Conserved residues (identical, upper case letters; similar, lower case letters) in all six proteins have a black background.

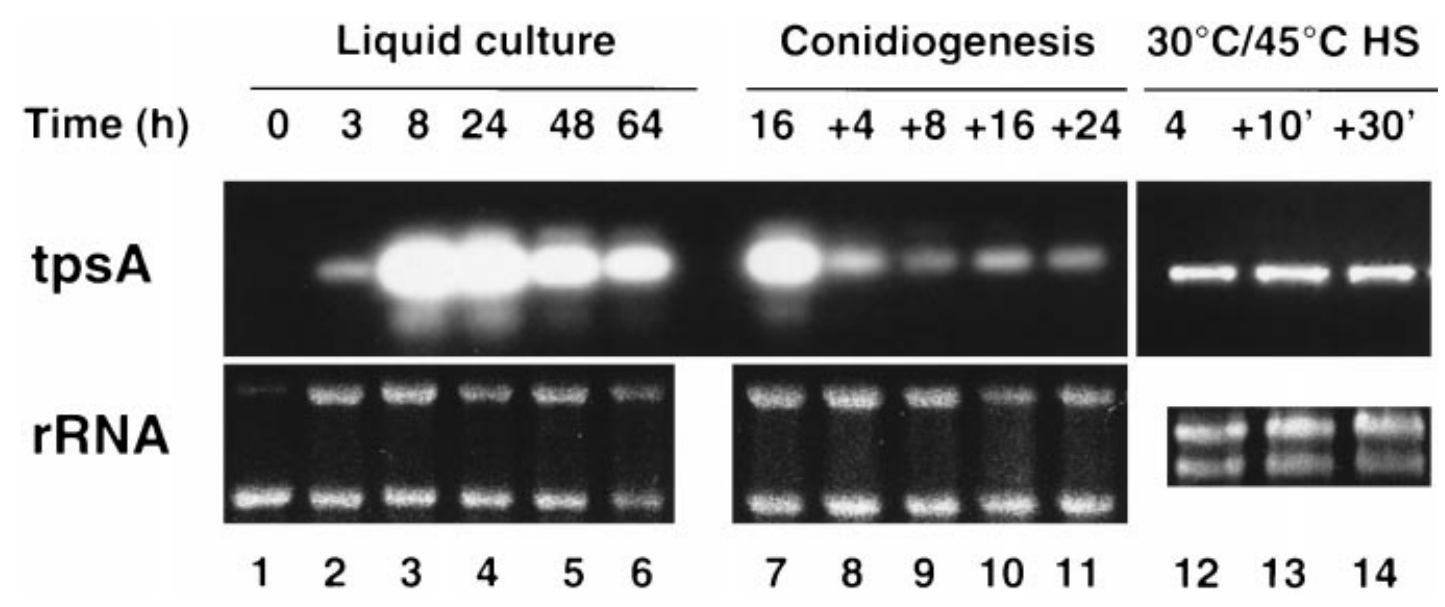

Fig. 3. Expression of the tpsA gene under different growth conditions. RNA was prepared from $A$. nidulans cultures grown under the following conditions: rich liquid medium for 0-64 $\mathrm{h}$ (samples 1-6); rich liquid medium for $16 \mathrm{~h}$ and transfer to rich solid medium for $0-24 \mathrm{~h}$ (samples 7-11); minimal glucose medium at $30^{\circ} \mathrm{C}$ for $4 \mathrm{~h}$ and transfer to $45{ }^{\circ} \mathrm{C}$ for 10 and $30 \mathrm{~min}$ (samples 12-14). Total RNA was reverse transcribed and subjected to an amplification using oligonucleotides that yield a PCR product specific to the tpsA transcript. Amplification was reduced to 20 cycles in order to obtain semi-quantitative data.

closest known homologue is the A. niger TpsA protein $(87 \cdot 4 \%$ identical amino acids and $90 \cdot 1 \%$ similar amino acids; Fig. 2).

\section{Regulation of the tpsA gene}

Expression of the A. nidulans tpsA gene under different culture conditions was monitored using semi-quantitative RT-PCR. Results presented in Fig. 3 show that transcription of the tps $A$ gene is induced during spore germination in rich medium (Fig. 3, lane 2), is maximal during exponential growth (Fig. 3, lanes 3-5 and lane 7) and decreases during the stationary phase of growth (Fig. 3, lane 6) and during conidiogenesis (Fig. 3, lanes 8-11). As a consequence, an RT-PCR product corresponding to the tpsA transcript could not be detected in non-germinating conidia of $A$. nidulans (Fig. 3, lane 1 ). When $4 \mathrm{~h}$ germlings of $A$. nidulans were exposed to 
Table 2. Trehalose levels in transformants of the S. cerevisiae tps $1 \Delta$ strain

\begin{tabular}{|llc|}
\hline Transforming plasmid & Cloned gene & $\begin{array}{c}\text { Trehalose } \\
\text { (\% of wet } \\
\text { weight })^{* *}\end{array}$ \\
\hline pCM190L & - & $0 \cdot 33$ \\
pCM190L::X & Control & $0 \cdot 31$ \\
pTPS16-1 & A. nidulans tpsA & $2 \cdot 10$ \\
pTPS16-2 & A. nidulans tpsA & $2 \cdot 13$ \\
\hline
\end{tabular}

* Trehalose levels in wild-type $S$. cerevisiae grown in minimal galactose medium are $1 \cdot 5-2 \cdot 0 \%$ of wet weight.

a heat shock at $45^{\circ} \mathrm{C}$ (Fig. 3, lanes 12-14) expression of tpsA was not altered.

\section{Expression of the tpsA gene complements the various defects of a $S$. cerevisiae tps $1 \Delta$ mutant}

S. cerevisiae strains lacking the TPS1 gene are deficient in the synthesis of trehalose in response to various stress conditions and are unable to grow on glucose or other rapidly fermentable sugars because of an uncontrolled influx of sugar into glycolysis, causing rapid ATP depletion (Van Aelst et al., 1993). To test whether tpsA could functionally complement the various defects of a $S$. cerevisiae tps $1 \Delta$ strain, the $t p s A$ ORF was amplified and cloned into the yeast expression vector pCM190L (Llorente et al., 1999), yielding plasmids pTPS16-1 and
pTPS16-2. After transformation of the plasmids into the yeast tps1 $1 \Delta$ mutant, growth of the transformants on glucose and galactose media was monitored. Expression of the A. nidulans tpsA gene fully restored growth of the $S$. cerevisiae tps $1 \Delta$ mutant on glucose (data not shown). Furthermore, trehalose production in the $S$. cerevisiae transformants expressing $A$. nidulans tps $A$ was restored to wild-type levels (Table 2). These results indicate that the A. nidulans TpsA protein can fulfil all of the functions of the yeast Tps1 T6PS, including the control of hexokinase function.

\section{Disruption of the tpsA gene in $A$. nidulans}

A pop-in/pop-out strategy was used to inactivate the tpsA gene. First, a strain carrying both the wild-type tps $A$ gene and a mutant allele separated by the $A$. fumigatus pyrG gene was constructed (CEA150: wA3 pyro A4 pyrG89 tpsA-pyrG-tpsAs) by transformation of A. nidulans strain FGSC773 with plasmid pTPS17. The tpsA $\Delta$ mutant allele removes 146 internal amino acids (90-235) in the 504 residue TpsA polypeptide, including stretches of amino acids that are highly conserved within fungal T6PS (Fig. 2). Excision of the A. fumigatus pyrG gene by recombination between the two $t p s A$ alleles was then forced in the presence of 5-FOA. PCR analysis of random 5-FOA-resistant clones using primers tps10 and tps11 or tps4 and tps5 (Table 1), which yield products of different sizes according to the tps $A$ allele, revealed that segregation between the wild-type and mutant alleles occurred randomly under these conditions (data not shown). Interestingly, all segregants with a tpsA $\Delta$ allele were thermosenitive for growth while the CEA150
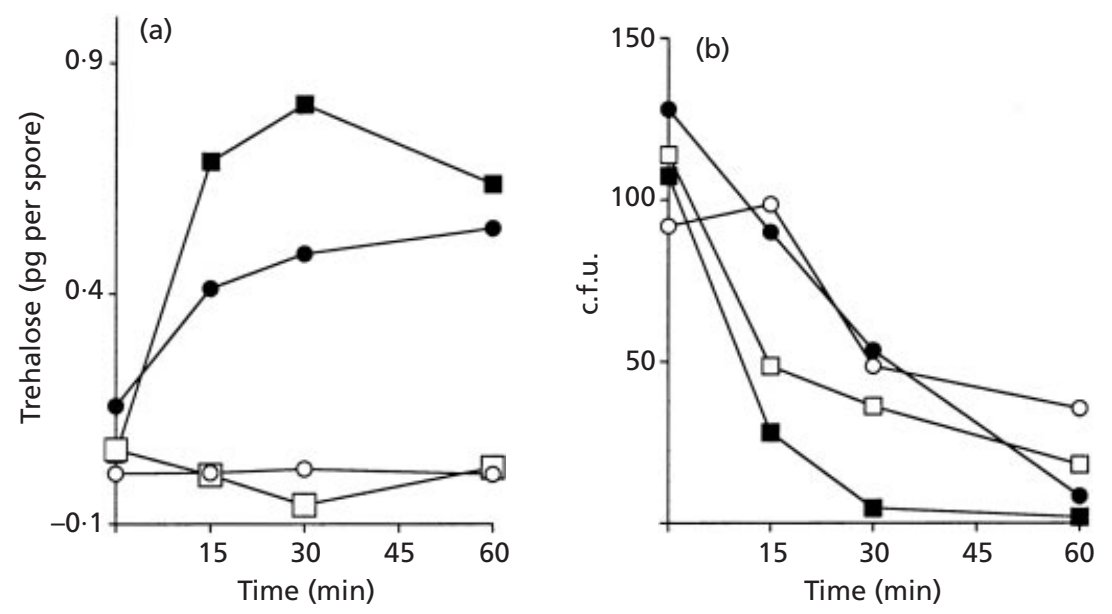

Fig. 4. A. nidulans tps $A \Delta$ mutants are defective for trehalose accumulation in response to a heat or oxidative stress. (a) Trehalose accumulation in response to a heat shock $\left(30^{\circ} \mathrm{C} \rightarrow 50^{\circ} \mathrm{C}\right)$ or an oxidative shock $\left(100 \mathrm{mM} \mathrm{H}_{2} \mathrm{O}_{2}\right)$ in germinating conidia of $A$. nidulans strains FGSC773 (wA3 pyroA4 pyrG89) ( $\mathbf{\square}$, heat shock; $\bullet$, oxidative shock) and CEA52 (wA3 pyroA4 pyrG89 tpsA $)\left(\square\right.$, heat shock; $\bigcirc$, oxidative shock). Conidia were germinated for $3 \mathrm{~h}$ in minimal glucose medium at $30{ }^{\circ} \mathrm{C}$ and subjected to the shock for the indicated times. (b) Conidia survival following a heat shock or an oxidative shock in germinating conidia of $A$. nidulans tps $A^{+}$and $\operatorname{tps} A \Delta$ strains. Duplicates of the samples analysed in (a) were diluted and plated on complete medium containing $0.01 \%$ Triton X-100. Colonies were counted after $2 \mathrm{~d}$ at $37^{\circ} \mathrm{C}$. Results are representative of two independent experiments. Symbols as in (a). 
Table 3. Metabolites accumulated in the wild-type and $\operatorname{tps} A \Delta$ strains during vegetative growth at $30^{\circ} \mathrm{C}$

Values are in $\mu \mathrm{mol}(\mathrm{g} \text { dry wt})^{-1}$ and are means of assays of two independent cultures and standard deviations are indicated. The ratios between values obtained for the mutant and wildtype strains are shown in parentheses.

\begin{tabular}{|lcc|}
\hline Metabolite & FGSC773 (wt) & $\begin{array}{c}\text { CEA152 } \\
(\boldsymbol{t} \boldsymbol{s} \boldsymbol{A \Delta})\end{array}$ \\
\hline Trehalose & $46 \cdot 5 \pm 2 \cdot 5$ & $<1 \cdot 0$ \\
Trehalose 6-phosphate & $2 \cdot 39 \pm 0 \cdot 65$ & $<0 \cdot 2$ \\
Glucose 6-phosphate & $0 \cdot 54 \pm 0 \cdot 10$ & $2 \cdot 49 \pm 0 \cdot 48(4 \cdot 6 \times)$ \\
Fructose 6-phosphate* & $0 \cdot 13$ & $0 \cdot 48(3 \cdot 7 \times)$ \\
Fructose 1,6-bisphosphate & $0 \cdot 33 \pm 0 \cdot 04$ & $0 \cdot 79 \pm 0.53(2 \cdot 4 \times)$ \\
ATP & $1 \cdot 37 \pm 0 \cdot 22$ & $2 \cdot 13 \pm 0 \cdot 42(1.5 \times)$ \\
\hline
\end{tabular}

*Values for fructose 6-phosphate were obtained from a single culture.

parental strain and $t p s A^{+}$progenies were not, suggesting that thermosensitive growth resulted from the inactivation of tpsA (see below). The occurrence of the tps $A \Delta$ allele in two of the 5-FOA-resistant clones was confirmed by Southern analysis of EcoRI/SalI-digested genomic DNA. One of the clones carrying the tpsA $\Delta$ allele was designated CEA152. A comparison of strains FGSC773 (pyrG89 wA3 pyroA4) and CEA152 (pyrG89 $w A 3$ pyroA4 tpsA $\Delta$ ) is reported below.

\section{tps $A$ is required for trehalose accumulation in response to various stress conditions}

In A. nidulans, trehalose is known to accumulate during conidiogenesis (d'Enfert \& Fontaine, 1997) as well as in response to heat shock (Noventa-Jordao et al., 1999). In other fungal species, trehalose has been shown to accumulate in the stationary phase of growth and in response to an oxidative or osmotic shock (Hounsa et al., 1998; Lewis et al., 1995; Lingappa \& Sussman, 1959; Van Laere, 1989; Wiemken, 1990). Accumulation of trehalose was therefore monitored in wild-type and tps $A \Delta$ conidia as well as in germinating conidia that were subjected to a heat shock or an oxidative shock. Trehalose could not be detected in mutant conidia (data not shown). When conidia of the wild-type strain FGSC773 were germinated for $3 \mathrm{~h}$ at $30^{\circ} \mathrm{C}$ and subsequently subjected to a $50{ }^{\circ} \mathrm{C}$ heat shock, a rapid increase in trehalose levels was observed (Fig. 4a).

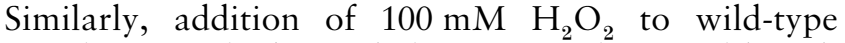
germlings resulted in trehalose accumulation, although to a lesser extent (Fig. 4a). Addition of $1 \mathrm{M} \mathrm{NaCl}$ did not result in a significant increase in trehalose levels (data not shown). In contrast to these results, neither heat shock nor $100 \mathrm{mM} \mathrm{H}_{2} \mathrm{O}_{2}$ resulted in trehalose accumulation in germlings of the tpsAs strain (Fig. 4a). Furthermore, T6P and trehalose were undetectable in the mycelium of a tpsA $\Delta$ strain (Table 3). We conclude that $t p s A$ encodes a T6PS essential for biosynthesis of T6P and trehalose in A. nidulans under various conditions, including conidiogenesis, heat shock and oxidative shock.

\section{Inactivation of tpsA does not increase sensitivity of germinating conidia to heat or oxidative shock}

It has previously been shown that viability of wild-type conidia is significantly decreased following a heat shock and that the loss of viability can be prevented by accumulated trehalose (d'Enfert et al., 1999). This would suggest that the inability to accumulate trehalose in response to stress may further increase the stress sensitivity of germinating conidia. To test this hypothesis, the viability of germinating wild-type or $t p s A \Delta$ conidia that had been subjected to a $50{ }^{\circ} \mathrm{C}$ heat shock or a $100 \mathrm{mM} \mathrm{H} \mathrm{O}_{2}$ shock (see above) was monitored. Results in Fig. 4(b) show that wild-type and tpsAs germlings display a similar sensitivity to heat and oxidative shock independent of the ability to accumulate trehalose. It therefore appears that the rapid accumulation of trehalose in response to a heat or oxidative stress does not contribute significantly to cell survival during short-term exposure to a stress.

\section{Inactivation of $\operatorname{tps} A$ results in thermosensitive growth}

To investigate the consequence of the inactivation of the tps A gene in A. nidulans, we first compared the growth of strains FGSC773 (wild-type) and CEA152 (tpsA $\Delta$ ) on various media and at different temperatures. The $t p s A \Delta$ mutant was unable to form colonies at temperatures above $44^{\circ} \mathrm{C}$ when glucose (Fig. 5a) or fructose (data not shown) were used as a carbon source. Thermosensitive growth was also observed when glucose was replaced by glycerol (Fig. 5a) although to a lesser extent. The tpsA $\Delta$ mutant also showed reduced growth on media containing sublethal doses (1-2 mM) of $\mathrm{H}_{2} \mathrm{O}_{2}$ (Fig. 5a).

Microscopic examination of conidia grown on minimal glucose medium revealed that the inability of strain CEA152 to establish a colony at temperatures above $44^{\circ} \mathrm{C}$ was due to a block of conidia germination (Fig. $5 \mathrm{~b}, \mathrm{c})$. Only a few spores had produced a germ tube after $24 \mathrm{~h}$ of growth at $44^{\circ} \mathrm{C}$ (Fig. $\left.5 \mathrm{c}\right)$. At lower temperatures, conidia of strain CEA152 were able to produce germ tubes at a frequency similar to that observed for the wild-type strain FGSC773, although with slower kinetics (Fig. 5b). When conidia were germinated on minimal glycerol medium at $45^{\circ} \mathrm{C}$ germination was only partially delayed (Fig. 5c).

Results presented in Fig. 6 show that the thermosensitive growth defect of strain CEA152 became irreversible after prolonged incubation at the non-permissive temperature and was limited to the developmental stages extending from spore germination to early filamentous growth. Indeed, when conidia of strain CEA152 were germinated at the non-permissive temperature $\left(45^{\circ} \mathrm{C}\right)$ 
(a)

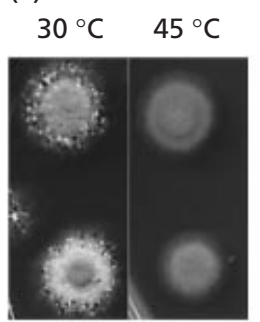

Glycerol

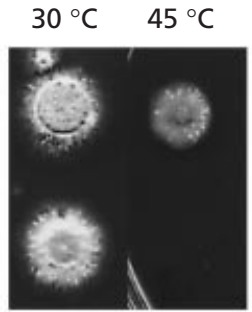

Glucose

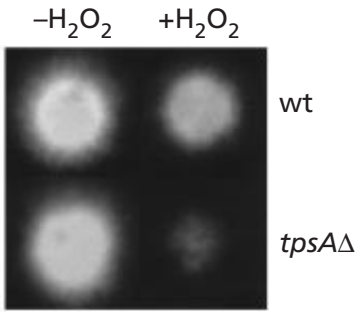

Glucose (c)
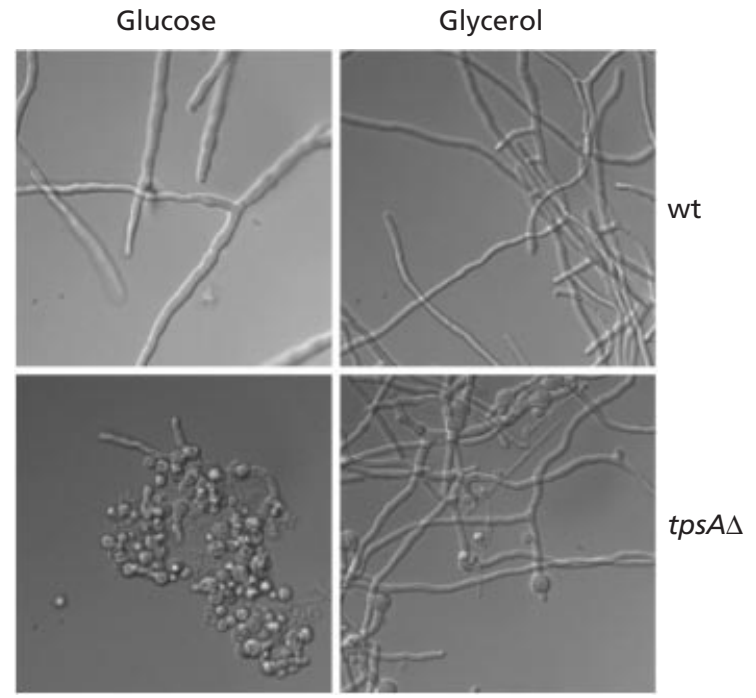

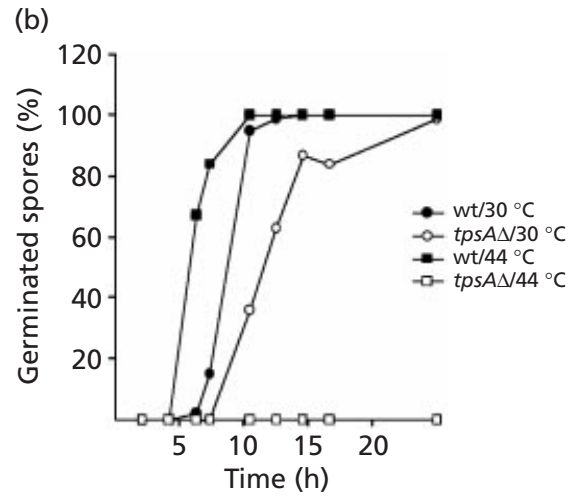

Fig. 5. Inactivation of tps $A$ results in a thermosensitive growth defect. (a) Comparison of the growth of the tps $A \Delta$ mutant (CEA152, wA3 pyroA4 pyrG89 tpsA $\Delta$ ) and the wild-type strain (FGSC773, wA3 pyroA4 pyrG89) on minimal medium with $1 \%$ glucose or $1 \%$ glycerol as carbon source in the presence or absence of $2 \mathrm{mM} \mathrm{H}_{2} \mathrm{O}_{2}$. Incubation was for $48-64 \mathrm{~h}$ at $30^{\circ} \mathrm{C}$ or $45^{\circ} \mathrm{C}$. (b) Germination frequency of the tps $A \Delta$ mutant and the wild-type strain at $30^{\circ} \mathrm{C}$ or $45^{\circ} \mathrm{C}$ in glucose-containing minimal medium. Results are representative of two independent experiments (c) Microscopic examination $(\times 570)$ of germinated spores after $24 \mathrm{~h}$ at $45^{\circ} \mathrm{C}$ in liquid minimal medium with the indicated carbon sources.

(a)

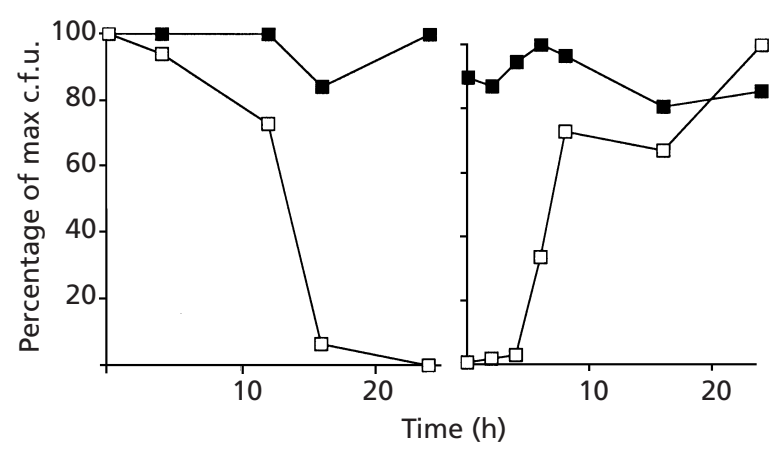

Fig. 6. The thermosensitive phenotype of the $\operatorname{tps} A \Delta$ mutant is dependent on the developmental stage. Shift experiments from restrictive $\left(45^{\circ} \mathrm{C}\right)$ to permissive $\left(37^{\circ} \mathrm{C}\right)$ temperature (a) and vice versa (b). Between 100 and 200 spores of the $A$. nidulans wildtype (FGSC773, wA3 pyroA4 pyrG89; $\mathbf{\square})$ and tpsAS (CEA152, wA3 pyroA4 pyrG89 tpsA $; \square$ ) strains were inoculated on complete medium and incubated for the indicated time periods before the shift. Counts represent the percentage of the maximal count. Results are representative of two independent experiments.

and shifted to the permissive temperature $\left(37^{\circ} \mathrm{C}\right)$ after various times, they were only able to form a colony when incubation at the non-permissive temperature was restricted to $12 \mathrm{~h}$ (Fig. 6a). In contrast, transfer to the non-permissive temperature of conidia germinated at the permissive temperature did not block colony formation when the transfer was performed after $10-12 \mathrm{~h}$ of germination (Fig. 6b).

\section{Inactivation of tpsA results in reduced conidium viability during prolonged storage}

Since the results presented above appeared to confirm a protective role of trehalose against sustained exposure of $A$. nidulans cells to various stress conditions, we evaluated the role of trehalose for the survival of conidia of A. nidulans during prolonged storage. Conidia of strains FGSC773, which contain trehalose, and CEA152, which are devoid of trehalose (data not shown), were maintained at $4{ }^{\circ} \mathrm{C}$ or $20^{\circ} \mathrm{C}$ for several weeks and the number of viable spores was estimated at different times. Results presented in Fig. 7 show that the conidia of the tps $A \Delta$ mutant strain lost viability much more rapidly than wild-type conidia when incubated at $20^{\circ} \mathrm{C}$. In contrast, no difference in viability between the wild-type and mutant conidia was observed upon incubation at $4{ }^{\circ} \mathrm{C}$ over a period of up to 7 weeks (Fig. 7). These results indicate that trehalose might play an important role in the survival of conidia during prolonged storage, either as a protective molecule or reserve carbohydrate. 


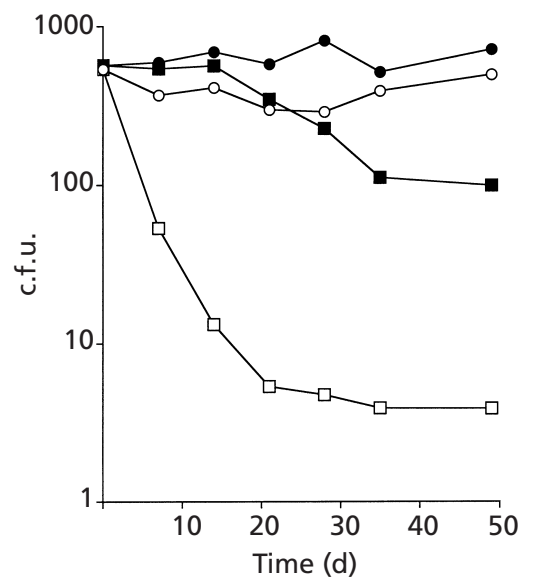

Fig. 7. Trehalose is required for conidium viability during prolonged storage. Viability of conidia from $A$. nidulans tps $A^{+}$ (FGSC773, wA3 pyroA4 pyrG89) or tpsA $\triangle$ (CEA152, wA3 pyroA4 pyrG89 tps $A \Delta$ ) strains upon prolonged incubation at $4{ }^{\circ} \mathrm{C}\left[\operatorname{tps} A^{+}\right.$ (O) and $\operatorname{tps} A \Delta(O)$, or $20^{\circ} \mathrm{C}, \operatorname{tps} A^{+}(\square)$ and $\left.\operatorname{tps} A \Delta(\square)\right]$. Conidia were resuspended in PBS/0.1\% Tween-20 at a density of $10^{9}$ conidia $\mathrm{ml}^{-1}$ and maintained at room temperature or $4{ }^{\circ} \mathrm{C}$ for the indicated times. Spore viability was determined by the number of c.f.u. after plating on complete medium and growth at $37^{\circ} \mathrm{C}$.

\section{DISCUSSION}

In this paper we have reported the cloning of the $A$. nidulans tpsA gene and physiological characterization of the tpsA $\Delta$ mutant. Our results demonstrate unambiguously that the tps $A$ gene product is a T6PS that is essential for trehalose biosynthesis in $A$. nidulans. The finding that tps $A$ is the unique T6PS-encoding gene in $A$. nidulans, in contrast to the situation in A. niger, where two T6PS genes have been identified (Wolschek \& Kubicek, 1997), and that inactivation of tpsA results in a complete loss of trehalose biosynthesis, in contrast to the inactivation of the $A$. nidulans T6PP-encoding orlA gene (Borgia et al., 1996), has allowed us for the first time to investigate the physiological role of trehalose in a filamentous fungus.

In the yeast $S$. cerevisiae, trehalose is synthesized by a large multi-subunit complex (Bell et al., 1998). The subunits of this complex share a domain which is similar to T6PS although devoid of T6PS activity. Our results show that the A. nidulans T6PS can fulfil all of the functions of its yeast counterpart, thus suggesting that it can associate in the multi-subunit trehalose synthase complex in yeast. Our reinvestigation of the sequence of the orlA gene shows that the A. nidulans T6PP is larger than previously proposed (Borgia et al., 1996) with an amino-terminal domain which is homologous to $A$. nidulans T6PS (C. d'Enfert \& A. Antczak, unpublished data). Furthermore, cDNAs encoding a homologue of $S$. cerevisiae Tps 3 have been identified in the course of expressed sequence tag (EST) sequencing of $A$. nidulans cDNAs (D. Kupfer \& B. Roe, http://www.genome. ou.edu/fungal.html; C. d'Enfert, unpublished data) thus suggesting that the $A$. nidulans trehalose synthase is also present in a multi-subunit complex in A. nidulans.

In $A$. nidulans trehalose has been shown to accumulate under a variety of conditions including nutrient starvation, conidiospore differentiation and heat stress (d'Enfert \& Fontaine, 1997; Noventa-Jordao et al., 1999; this study). Here we have shown that trehalose accumulation is also stimulated in response to an oxidative stress (Fig. 4a) consistent with the results of Noventa-Jordao et al. (1999), who showed a link between heat shock recovery and the cellular response to oxidative stress in A. nidulans. Results presented in this paper show that trehalose accumulation is mediated by TpsA under the different conditions tested, i.e. heat stress, oxidative stress and conidiogenesis. Furthermore, TpsA is responsible for the basal levels of trehalose and T6P that are produced during mycelial growth (data not shown). Although these results would suggest that trehalose biosynthesis in response to temperature and oxidative stress could contribute to the resistance of the germlings to these stress conditions, results presented in Fig. 4(b) show that this is not always the case: wild-type and tpsA-null germlings are similarly sensitive to a heat or an oxidative shock. In contrast, the absence of trehalose and/or trehalose biosynthesis results in reduced spore viability and a reduced ability to grow upon constant exposure to sublethal stress, including prolonged exposure to high temperature and growth in the presence of reactive oxygen species. Taken together, these results suggest that trehalose is mainly involved in the resistance of $A$. nidulans to progressive exposure to lethal stress or prolonged exposure to sublethal stress rather than rapid exposure to lethal stress. This is in agreement with the previous observations that germlings of $A$. nidulans maintaining a high level of trehalose due to a defect in the TreB neutral trehalase are less sensitive to heat stress than wild-type germlings (d'Enfert et al., 1999) and that viability of the conidia of an A. niger tpsA mutant is reduced (Wolschek and Kubicek, 1997). This is also consistent with the role of trehalose in the acquisition of thermotolerance and halotolerance demonstrated in S. cerevisiae (Hounsa et al., 1998; Lewis et al., 1995) and in Schizosaccharomyces pombe (Ribeiro et al., 1998). Results presented in Fig. 6 show that this protective role of trehalose is most important during $A$. nidulans conidial germination as opposed to later developmental stages, including mycelial growth, suggesting that additional mechanisms of adaptation to stress operate following germ tube formation.

Analysis of the expression of the tpsA gene in response to a heat shock or during conidiogenesis, when trehalose is synthesized, did not reveal induction of the transcription of tpsA under these conditions (Fig. 3). In contrast, expression of $t p s A$ is induced during the early stages of mycelial growth, when trehalose biosynthesis appears minimal. Our results contrast with those of Wolschek \& Kubicek (1997), who showed that the A. niger tps $A$ and $t p s B$ genes are respectively downregulated and up-regulated by a heat shock. While 
activation of trehalose biosynthesis in A. niger appears to be controlled in part at the transcriptional level, our data suggest that, in A. nidulans, an inactive form of T6PS is accumulated during phases of rapid growth to prepare for induction of trehalose biosynthesis in response to stress, nutrient starvation, or developmental transitions by means of post-transcriptional control mechanisms. In S. cerevisiae T6PS activation is mediated both at the transcriptional level through an STREdependent activation mechanism (de Virgilio et al., 1993; Winderickx et al., 1996) and at the post-translational level by the protein kinase Rim15 (Reinders et al., 1998). A homologue of Rim15 has been identified by systematic sequencing of $A$. nidulans cDNAs (D. Kupfer $\&$ B. Roe, http://www.genome.ou.edu/fungal.html; C. d'Enfert, unpublished data). It is a possible candidate for post-translational control of TpsA, allowing rapid activation of trehalose synthesis under stress conditions.

In $S$. cerevisiae, inactivation of the T6PS results in an inability to grow on rapidly fermentable sugars such as glucose and fructose because of an uncontrolled influx of the sugars into glycolysis, causing rapid ATP depletion (Van Aelst et al., 1993). Recent results suggest that both T6P inhibition of hexokinase and a direct involvement of T6PS are responsible for this phenomenon (Bonini et al., 2000). In A. nidulans, T6P is also known to inhibit hexokinase (Ruijter et al., 1996) and our results show that TpsA is able to fulfil all the functions of the yeast T6PS, including its control on glucose influx into glycolysis, thus suggesting that similar mechanisms of glucose influx could operate in yeast and $A$. nidulans. On the other hand, the $A$. nidulans tpsA-null mutant is able to grow on glucose or fructose as a carbon source. Although increased levels of sugar phosphates could be detected in the mycelium of the mutant strain grown at $30^{\circ} \mathrm{C}$, this increase was not associated with a decrease of the ATP pool (Table 3). This suggests that T6P is a physiological inhibitor of hexokinase in $A$. nidulans but that the increase in glycolytic flux resulting from T6P depletion has only minor consequences in this fungus compared to what has been observed in S. cerevisiae. This physiological role of T6P is also supported by the poor growth on fructose of the A. nidulans orlA mutant which accumulates T6P and consequently should have reduced hexokinase activity (S. Fillinger \& C. d'Enfert, unpublished results). Interestingly, the thermosensitive growth defect of the $A$. nidulans tps $A \Delta$ strain appeared less pronounced on minimal glycerol medium than on minimal glucose medium (Fig. 5). Although this may reflect a more stringent role of T6P on the control of glycolytic flux at high temperature or the replacement of trehalose as a stress metabolite by intracellular glycerol resulting from glycerol uptake, the thermosensitive growth defect might also be less pronounced on slowly metabolizable carbon substrate because of a slower growth rate and the resulting presence of a higher intrinsic stress resistance (see Thevelein \& de Winde, 1999, for a recent review). Further analysis of metabolic fluxes at different temperatures in wild-type and tre- halose biosynthesis mutants is needed to assess precisely the role of T6P glycolytic control in filamentous fungi.

In summary, results presented in this study show that $\mathrm{T} 6 \mathrm{P}$, in addition to its role in the control of chitin biosynthesis (Borgia et al., 1996), appears to play only a minor role in the control of the glycolytic flux in $A$. nidulans, in contrast to what has been observed in $S$. cerevisiae and some phylogenetically close yeast species (Kluyveromyces lactis, Candida albicans). More importantly, our results show that trehalose is a major stress metabolite in A. nidulans and is probably involved in the acquisition of resistance to a variety of stress conditions, including heat and oxidative stress, as well as in the survival of conidia during prolonged storage.

\section{ACKNOWLEDGEMENTS}

We are indebted to Jaap Visser for his constant support and interest during the course of this work. Thanks are due to Jean Paul Latgé, in whose laboratory part of this work was carried out, to Markus Wolschek and Christian P. Kubicek from Section Microbial Biochemistry, Institute for Biochemical Technology and Microbiology, TU Wien, Austria, for their contribution to the sequencing of the $A$. nidulans tps $A$ gene and to Bertrand Llorente for providing plasmid pCM190L. This work was supported by the EU Biotechnology Programme (BIO4-CT98-0268).

\section{REFERENCES}

Arguelles, J. C. (2000). Physiological roles of trehalose in bacteria and yeasts: a comparative analysis. Arch Microbiol 174, 217-224.

Arisan-Atac, I., Wolschek, M. F. \& Kubicek, C. P. (1996). Trehalose6-phosphate synthase A affects citrate accumulation by Aspergillus niger under conditions of high glycolytic flux. FEMS Microbiol Lett 140, 77-83.

Ausubel, F. M., Brent, R., Kingston, R. E., Moore, D. D., Seidman, J. G., Smith, J. A. \& Struhl, K. (1992). Short Protocols in Molecular Biology. New York: Wiley.

Bell, W., Klaassen, P., Ohnacker, M., Boller, T., Herweijer, M., Schoppink, P., Van der Zee, P. \& Wiemken, A. (1992). Characterization of the $56-\mathrm{kDa}$ subunit of yeast trehalose-6-phosphate synthase and cloning of its gene reveal its identity with the product of CIF1, a regulator of carbon catabolite inactivation. Eur J Biochem 209, 951-959.

Bell, W., Sun, W., Hohmann, S., Wera, S., Reinders, A., de Virgilio, C., Wiemken, A. \& Thevelein, J. M. (1998). Composition and functional analysis of the Saccharomyces cerevisiae trehalose synthase complex. J Biol Chem 273, 33311-33319.

Blazquez, M. A., Lagunas, R., Gancedo, C. \& Gancedo, J. M. (1993). Trehalose-6-phosphate, a new regulator of yeast glycolysis that inhibits hexokinases. FEBS Lett 329, 51-54.

Blazquez, M. A., Stucka, R., Feldmann, H. \& Gancedo, C. (1994). Trehalose-6-P synthase is dispensable for growth on glucose but not for spore germination in Schizosaccharomyces pombe. J Bacteriol 176, 3895-3902.

Bonini, B. M., Van Vaeck, C., Larsson, C., Gustafsson, L., Ma, P., Winderickx, J., Van Dijck, P. \& Thevelein, J. M. (2000). Expression of Escherichia coli ots A in a Saccharomyces cerevisiae tps1 mutant restores trehalose 6-phosphate levels and partly restores growth and fermentation with glucose and control of glucose influx into glycolysis. Biochem J 350, 261-268. 
Borgia, P. T., Miao, Y. \& Dodge, C. L. (1996). The orlA gene from Aspergillus nidulans encodes a trehalose-6-phosphate phosphatase necessary for normal growth and chitin synthesis at elevated temperatures. Mol Microbiol 20, 1287-1296.

Brody, H., Griffith, J., Cuticchia, A. J., Arnold, J. \& Timberlake, W. E. (1991). Chromosome-specific recombinant DNA library from the fungus Aspergillus nidulans. Nucleic Acids Res 19, 3105-3109.

Chen, D. C., Yang, B. C. \& Kuo, T. T. (1992). One-step transformation of yeast in stationary phase. Curr Genet 21, 83-84.

Devereux, J., Haeberli, P. \& Smithies, O. (1984). A comprehensive set of sequence analysis programs for the VAX. Nucleic Acids Res 12, 387-395.

Elbein, A. D. (1974). The metabolism of $\alpha, \alpha$-trehalose. $A d v$ Carbohydr Chem Biochem 30, 256-277.

d'Enfert, C. (1996). Selection of multiple disruption events in Aspergillus fumigatus using the orotidine-5'-decarboxylase gene, pyrG as a unique transformation marker. Curr Genet 30, 76-82.

d'Enfert, C. \& Fontaine, T. (1997). Molecular characterization of the Aspergillus nidulans tre A gene encoding an acid trehalase required for growth on trehalose. Mol Microbiol 24, 203-216.

d'Enfert, C., Bonini, B. M., Zapella, P. D. A., Fontaine, T., da Silva, A. M. \& Terenzi, H. F. (1999). Neutral trehalases catalyse intracellular trehalose breakdown in the filamentous fungi Aspergillus nidulans and Neurospora crassa. Mol Microbiol 32, 471-484.

Girardin, H., Latgé, J.-P., Skirantha, T., Morrow, B. \& Soll, D. (1993). Development of DNA probes for fingerprinting Aspergillus fumigatus. J Clin Microbiol 31, 1547-1554.

Hanahan, D., Jessee, J. \& Bloom, F. R. (1991). Plasmid transformation of Escherichia coli and other bacteria. Methods Enzymol 204, 63-113.

Hohmann, S., Bell, W., Neves, M. J., Valckx, D. \& Thevelein, J. M. (1996). Evidence for trehalose-6-phosphate-dependent and -independent mechanisms in the control of sugar influx into yeast glycolysis. Mol Microbiol 20, 981-991.

Hounsa, C.-G., Vincent, E., Thevelein, J. M., Hohmann, S. \& Prior, B. (1998). Role of trehalose in survival of Saccharomyces cerevisiae under osmotic stress. Microbiology 144, 671-680.

Kopp, M., Müller, H. \& Holzer, H. (1993). Molecular analysis of the neutral trehalase gene from Saccharomyces cerevisiae. J Biol Chem 268, 4766-4774.

Lewis, J. G., Learmonth, R. P. \& Watson, K. (1995). Induction of heat, freezing and salt tolerance by heat and salt shock in Saccharomyces cerevisiae. Microbiology 141, 687-694.

Lingappa, B. T. \& Sussman, A. S. (1959). Endogenous substrates of dormant, activated and germinating ascospores of Neurospora tetrasperma. Plant Physiol 34, 466-473.

Llorente, B., Fairhead, C. \& Dujon, B. (1999). Genetic redundancy and gene fusion in the genome of the baker's yeast Saccharomyces cerevisiae: functional characterization of a three-member gene family involved in the thiamine biosynthetic pathway. Mol Microbiol 32, 1140-1152.

Luyten, K., de Koning, W., Tesseur, I., Ruiz, M. C., Ramos, J., Cobbaert, P., Thevelein, J. M. \& Hohmann, S. (1993). Disruption of the Kluyveromyces lactis GGS1 gene causes inability to grow on glucose and fructose and is suppressed by mutations that reduce sugar uptake. Eur J Biochem 217, 701-713.

Mol, P. C., Mouyna, I. \& d'Enfert, C. (1998). A rapid procedure for the extraction of genomic DNA from intact Aspergillus spores. Fungal Genet Newsl 45, 28-29.
Neves, M. J., Terenzi, H. F., Leone, F. A. \& Jorge, J. A. (1994). Quantification of trehalose in biological samples with a conidial trehalase from the thermophilic fungus Humicola grisea var. thermoidea. World J Microbiol Biotechnol 10, 17-19.

Neves, M. J., Hohmann, S., Bell, W. \& 7 other authors (1995). Control of glucose influx into glycolysis and pleiotropic effects studied in different isogenic sets of Saccharomyces cerevisiae mutants in trehalose biosynthesis. Curr Genet 27, 110-122.

Noventa-Jordao, M. A., Couto, R. M., Goldman, M. H., Aguirre, J., lyer, S., Caplan, A., Terenzi, H. F. \& Goldman, G. H. (1999). Catalase activity is necessary for heat-shock recovery in Aspergillus nidulans germlings. Microbiology 145, 3229-3234.

Nwaka, S., Mechler, B., Destruelle, M. \& Holzer, H. (1995). Phenotypic features of trehalase mutants in Saccharomyces cerevisiae. FEBS Lett 360, 286-290.

Osmani, S. A., May, G. S. \& Morris, R. N. (1987). Regulation of the mRNA levels of nimA, a gene required for the G2-M transition in Aspergillus nidulans. J Cell Biol 104, 1495-1504.

Panneman, H., Ruijter, G. J. G., van den Broeck, H. C. \& Visser, J. (1998). Cloning and biochemical characterization of Aspergillus niger hexokinase. The enzyme is strongly inhibited by physiological concentrations of trehalose-6-phosphate. Eur J Biochem 258, 223-232.

Prade, R. A., Griffith, J., Kochut, K., Arnold, J. \& Timberlake, W. E. (1997). In vitro reconstruction of the Aspergillus (=Emericella) nidulans genome. Proc Natl Acad Sci US A 94, 14564-14569.

Reinders, A., Burckert, N., Hohmann, S., Thevelein, J. M., Boller, T., Weimken, A. \& De Virgilio, C. (1997). Structural analysis of the subunits of the trehalose-6-phosphate synthase/phosphatase complex in Saccharomyces cerevisiae and their function during heat shock. Mol Microbiol 24, 687-695.

Reinders, A., Burckert, N., Boller, T., Wiemken, A. \& De Virgilio, C. (1998). Saccharomyces cerevisiae cAMP-dependent protein kinase controls entry into stationary phase through the Rim15p protein kinase. Genes Dev 12, 2943-2955.

Ribeiro, M. J. S., Reinders, A., Boller, T., Wiemken, A. \& de Virgilio, C. (1998). Trehalose synthesis is important for the acquisition of thermotolerance in Schizosaccharomyces pombe. Mol Microbiol 25, 571-581.

Ruijter, G. J. G. \& Visser, J. (1996). Determination of intermediary metabolites in Aspergillus niger. J Microbiol Methods 25, 295-302.

Ruijter, G. J. G., Panneman, H., van den Broeck, H. C., Bennett, J. M. \& Visser, J. (1996). Characterisation of the Aspergillus nidulans frA1 mutant: hexose phosphorylation and apparent lack of involvement of hexokinase in glucose repression. FEMS Microbiol Lett 139, 223-228.

Sambrook, J., Fritsch, E. F. \& Maniatis, T. (1989). Molecular Cloning: a Laboratory Manual. Cold Spring Harbor, NY: Cold Spring Harbor Laboratory.

Sanger, F., Nicklen, S. \& Coulson, R. A. (1977). DNA sequencing with chain terminating inhibitors. Proc Natl Acad Sci USA 74, 5463-5467.

Sherman, F. (1991). Getting started with yeast. Methods Enzymol 194, 3-21.

Thevelein, J. M. (1984). Regulation of trehalose mobilization in fungi. Microbiol Rev 48, 42-59.

Thevelein, J. M. (1996). Regulation of trehalose metabolism and its relevance to cell growth and function. In The Mycota III. Biochemistry and Molecular Biology, pp. 395-420. Edited by R. Brambl \& G. A. Marzluf. Berlin: Springer. 
Thevelein, J. M. \& Hohmann, S. (1995). Trehalose synthase: guard to the gate of glycolysis in yeast. Trends Biochem Sci 20,3-9.

Thevelein, J. M. \& de Winde, J. H. (1999). Novel sensing mechanisms and targets for the cAMP-protein kinase A pathway in the yeast Saccharomyces cerevisiae. Mol Microbiol 33, 904-918.

Thomas, B. J. \& Rothstein, R. (1989). Elevated recombination rates in transcriptionally active DNA. Cell 56, 619-630.

Van Aelst, L., Hohmann, S., Bulaya, B. \& 7 other authors (1993). Molecular cloning of a gene involved in glucose sensing in the yeast Saccharomyces cerevisiae. Mol Microbiol 8, 927-943.

Van Laere, A. (1989). Trehalose, reserve and/or stress metabolite? FEMS Microbiol Rev 63, 201-210.

de Virgilio, C., Bückert, N., Bell, W., Jenö, P., Boller, T. \& Wiemken, A. (1993). Disruption of TPS2, the gene encoding the $100 \mathrm{kDa}$ subunit of the trehalose-6-phosphate synthase/phosphatase complex in Saccharomyces cerevisiae, causes accumulation of trehalose-6-phosphate and loss of trehalose-6-phosphate phosphatase activity. Eur J Biochem 212, 315-323.

Vuorio, O. E., Kalkkinen, N. \& Londesborough, J. (1993). Cloning of two related genes encoding the $56-\mathrm{kDa}$ and $123-\mathrm{kDa}$ subunits of trehalose synthase from the yeast Saccharomyces cerevisiae. Eur J Biochem 216, 849-861.

Weidner, G., d'Enfert, C., Koch, A., Mol, P. C. \& Brakhage, A. A.
(1998). Development of a homologous transformation system for the pathogenic fungus Aspergillus fumigatus based on the pyrG gene encoding orotidine monophosphate decarboxylase. Curr Genet 33, 378-385.

Wiemken, A. (1990). Trehalose in yeast, stress protectant rather than reserve carbohydrate. Antonie Leeuwenhoek 58, 209-217.

Winderickx, J., de Winde, J. H., Crauwels, M., Hino, A., Hohmann, S., Van Dijck, P. \& Thevelein, J. M. (1996). Regulation of genes encoding subunits of the trehalose synthase complex in Saccharomyces cerevisiae: novel variations of STRE-mediated transcription control? Mol Gen Genet 252, 470-482.

Wolschek, M. F. \& Kubicek, C. P. (1997). The filamentous fungus Aspergillus niger contains two "differentially regulated" trehalose-6-phosphate synthase-encoding genes, tps $A$ and $t p s B$. $J$ Biol Chem 272, 2729-2735.

Woodcock, D. M., Crowther, P. J., Doherty, J., Jefferson, S., DeCruz, E., Noyer-Weidner, M., Smith, S. S., Michael, M. Z. \& Graham, M. W. (1989). Quantitative evaluation of Escherichia coli host strains for tolerance to cytosine methylation in plasmid and phage recombinants. Nucleic Acids Res 17, 3469-3478.

Received 29 December 2000; revised 15 March 2001; accepted 29 March 2001. 